\title{
Analytical Approach for the TH-BPSK Ultra-Wideband Systems Performance
}

\author{
Chihi Houda, Hizem Moez, and Bouallegue Ridha
}

\begin{abstract}
This paper characterizes the performance of Time Hopping Binary Phase-shift Keying (TH-BPSK) multiple access schemes for Ultra Wideband (UWB) communications by validating an analytical study based on the bit error probability (BER) evaluation versus signal to noise ratio (SNR). Analysis of TH-BPSK is assessed over the approach used in [1] for a Time Hopping Binary Pulse Position Ultra-Wideband Modulation scheme (TH BPPM-UWB) and a comparison is made between both. For communicating over an AWGN channel, the simulation results show that the BPSK modulation performance's is better than that of the PPM for all values of SNR.
\end{abstract}

Index Terms-UWB, performance, probability of error, TH-BPSK modulation, TH-PPM modulation.

\section{INTRODUCTION}

UWB is a novel technology emerging in recent years as a promising solution for high speed or low power indoor communications. Indeed, this technology offers the potential for robust communications in multipath and multi-user environments, as well as low cost and low complexity implementations. However, the performance of UWB systems is strongly dependant on the multiple accesses and used modulation techniques.

TH multiple access technique, where users are distinguished by their respective pulse arrival time sequences, is one of the most popular multiple access techniques used for an impulse radio system [2]. The combination between time hopping (TH) and pulse position modulation (PPM) is the original modulation type proposed for UWB systems [3], [4]. A performance study of this modulation in terms of BER has been mentioned in [5] with an AWGN channel.

Currently, TH-PPM and TH-BPSK UWB systems have the same interest despite the performance difference in terms of bit error rate (BER) especially. Analysis of TH-BPSK UWB systems was analyzed and studied in [6], [7], [8]. In [9], a performance comparison between different modulation schemes was applied. In this paper, our purpose is to provide an analytical method to evaluate the BER performance of TH-BPSK with the presence of an AWGN channel.

This paper is organized as follows. Section II describes the TH-BPSK UWB system model. In Section III, we elaborate an analytical approach to determine the performance of this modulation scheme and then, we validate the theoretical analysis with simulation results. In Section IV, we compare

Manuscript received August 1, 2012; revised October 1, 2012.

C. Houda and H. Moez are with the National Engineer School of Tunis (ENIT), Tunisia (e-mail: houda.chihi@gmail.com, moezhizem@yahoo.fr).

B. Ridha is with the Higher School of Communication of Tunis (Sup'COM), Tunisia (e-mail: idha.bouallegue@supcom.rnu.tn). the performances of TH-BPSK and TH-PPM UWB system models using our analytical analysis. Finally, some conclusions are given in Section V.

\section{TH-BPSK UWB SYSTEM MODEL}

In general, the UWB system is formed by short pulses. For the BPSK case, the information depends on the pulses polarities, if $d_{j}$ is the source signal and $\beta_{i}$ the BPSK signal modulation. This is presented as follows [8],

$$
\beta_{\varphi}=2 \times d j-1= \begin{cases}-1, & d j=0 \\ +1, & d j=1\end{cases}
$$

From (1), we note that the basic pulse sign's of TH-BPSK is modulated according to the data bit where for each user is assigned an unique time hopping random sequence. In our case, we consider a TH-BPSK-UWB system to apply our analytical approach. A typical transmitted TH-BPSK signal model is given in Fig. 1 [10].

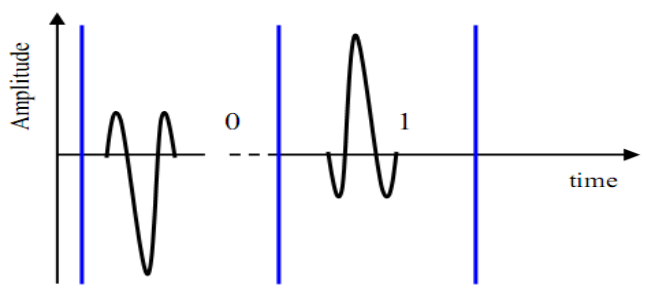

Fig. 1. TH-BPSK UWB signal model.

This modulation type can be modeled as follows for the $\mathrm{m}^{\text {th }}$ user [8],

$$
\mathrm{S}_{\mathrm{BPSK}}{ }^{(k)}(t, i)=\sqrt{\frac{E_{b}}{N_{S}}} \sum_{j=i N_{S}}^{(i+1) N_{s}-1} d_{i}{ }^{k} p\left(t-j T_{f}-c_{j}{ }^{(k)} T_{c}\right)
$$

where $\mathrm{s}_{\mathrm{BPSK}}{ }^{(k)}(t, i)$ is the signal transmitted by one user corresponding to the $\mathrm{i}^{\text {th }}$ data bit and $p(t)$ is the transmitted UWB pulse shape. $E_{b}$ is the total energy of one information bit. $T_{f}$ is the duration of a frame which can be subdivided into chips of duration $T_{c} . c_{j}^{(k)} \in\left\{0,1, \ldots, N_{h}-1\right\}$ is the TH sequence related to the $\mathrm{k}^{\text {th }}$ source where $N_{h}$ represents the number of hops and satisfies $N_{h} T_{c} \leq T_{f}$ [11]. As TH-BPSK UWB modulation is antipodal, $d_{i}^{(k)} \in\{1,-1\}$ represents the $i^{\text {th }}$ data bit transmitted by the $k^{\text {th }}$ source with the same probability.

\section{ANALYTICAL APPROACH FOR TH-BPSK UWB SYSTEM MODEL}

The performance analysis starts by studying the reception of the TH-BPSK system. If we consider the TH-BPSK performance for one user and one path, (2) becomes, 


$$
S_{B P S K}(t, i)=\sqrt{\frac{E_{b}}{N_{S}}} \sum_{j=i N_{S}}^{(i+1) N_{s}-1} d_{i} p\left(t-j T_{f}-c_{j} T_{c}\right)
$$

If we suppose that the temporal support is disjoints, we obtain after calculation (4),

$$
d_{i}=2 \frac{E_{b}}{N_{s}}\left(N_{s}-1\right) d_{i} R_{w}+\widetilde{b}
$$

With $R_{w}=\int_{[T]} p^{2}(t) d t$ represents the autocorrelation function of the transmitted signal , and $\tilde{b}$ determines the noise power. In the noise less case $(b(t)=0)$, we obtain,

$$
\begin{gathered}
d_{i}=1 ; D_{i}=\frac{E_{b}}{N_{s}}\left(N_{s}-1\right) R_{w}>0 \\
d_{i}=-1 ; D_{i}=-\frac{E_{b}}{N_{s}}\left(N_{s}-1\right) R_{w}<0
\end{gathered}
$$

The performance study of TH-BPSK is based on the comparison of the received sequences with the emitted ones. If we suppose that the $d_{i}$ are equiprobably distributed, we have then,

$$
P_{e}=\operatorname{Prob}\left(\hat{d}_{i} \neq d_{i} / d_{i}=1\right)=\operatorname{Prob}\left(D_{i}<0 / d_{i}=1\right)
$$

After calculation, we find the expression of the error probability,

$$
P_{e}=\operatorname{Prob}\left(\frac{\tilde{b}}{N_{0}}>2 \frac{E_{b}}{N_{0}} R_{w}\right)
$$

To determine the autocorrelation function, we consider the Gaussian impulse relation as follows,

$$
w_{t r}(t)=\left(\frac{t}{\tau}\right) e^{\left(-\left(\frac{\mathrm{t}}{\tau}\right)\right)^{2}}
$$

Consequently, the autocorrelation function $R_{w}$ becomes,

$$
R_{w}(0)=\tau \frac{\sqrt{2 \pi}}{8}
$$

By following this way and replacing $R_{w}(0)$ by its expression, we obtain the equation (9), which represents the performance of TH-BPSK UWB system model for one user and one path,

$$
P_{e}=Q\left(\tau \frac{\sqrt{2 \pi}}{8} \sqrt{\frac{2 E_{b}}{N_{0}}}\right)
$$

Which $E_{b}$ represents the bit energy, $N_{0}$ is the power noise and $Q$ is the Marcum function.

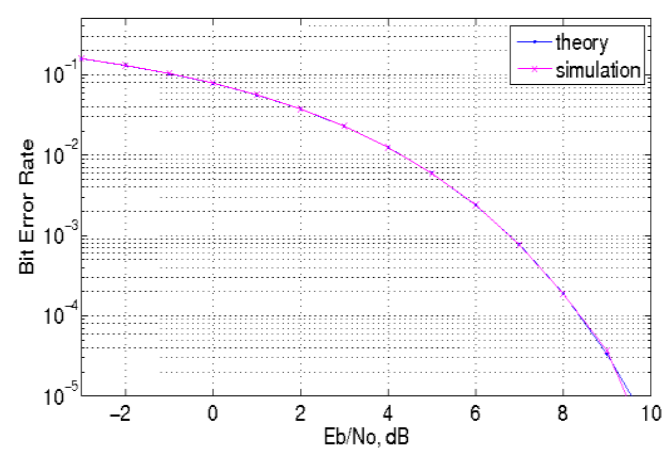

Fig. 2 . Comparison between analytical and simulation results for TH-BPSK UWB systems.

Fig. 2 shows BER curve of TH-BPSK system in theory case versus simulation with the MATLAB tool. We note, from this figure, that the theoretical and simulated results are approximately similar. This validates the approach using our new analytical analysis.

\section{PERFORMANCE COMPARISON BETWEEN TH-BPSK AND TH-PPM UWB SYSTEM MODEL}

\section{A. TH-PPM UWB System Model}

TH-PPM UWB system model is the very popular scheme because it depends to the pulse that will be sent early or late relatively to the data transmitted. In [11], PPM is defined as a modulation type that introduces a shift $(\delta)$ to distinguish between the pulses related to the bit " 0 " and the bit " 1 ".

A typical UWB TH-PPM waveform can be modeled as follows,

$$
\sum_{i=0} \sum_{j=0}^{N_{s}-1} w_{t r}\left(t-i T_{s}-j T_{f}-c_{j} T_{c}-d_{i} \delta\right)
$$

where $w_{t r}$ is the transmitted pulse shape of duration $T_{p} . T_{s}$ is the symbol duration. $T_{f}$ is the frame period. $N_{s}$ is the chips number with duration $T_{c}$ and $d_{i} \in\{0,1\}$ is the data bit transmitted.

After calculation of the probability of error as the same method as TH-BPSK UWB system model, we obtain the equation (10) related to the performance of TH-PPM UWB system model found in [1],

$$
P_{e}=\mathrm{Q}\left(S N R \cdot\left[1-\tau \cdot\left(\exp \left(-\frac{\sigma^{2}}{2 \tau^{2}}\right)\right) \cdot\left(1-\frac{\sigma^{2}}{\tau^{2}}\right)\right]\right)
$$

Fig. 3 shows the performance of TH-PPM UWB system model found in the theoretical case from (10) compared to the simulation results.

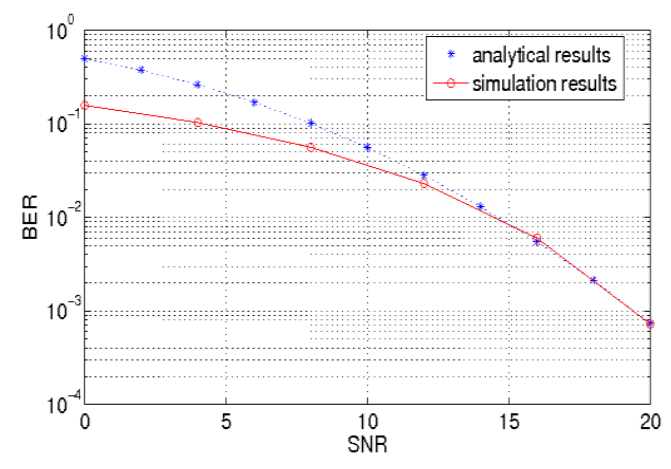

Fig. 3. TH-PPM UWB system model performance with our analytical approach

\section{B. Performance Comparison}

In this part, we compare the performance between the TH-BPSK and TH-PPM UWB system models with the results found in (8) and (10). This is given in Fig. 4, which represents the comparison performance between TH-BPSK and TH-PPM system models with our analytical approach.

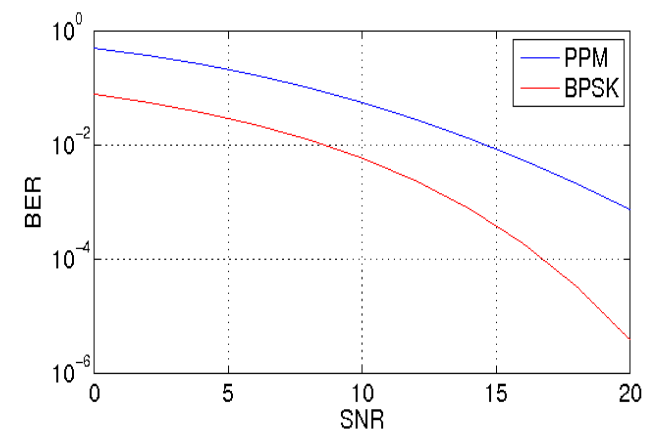

Fig. 4. Comparison performance between TH-BPSK and TH-PPM UWB system models with our analytical approach.

From this figure, we notice that the UWB TH-BPSK system model is better than TH-PPM for all values of SNR. 
This further validates our analytical approach, as confirmed by other previous studies in this domain [7].

While TH-BPSK is antipodal, this modulation scheme agrees with the proprieties of UWB system which consequently is better than TH-PPM that's an orthogonal signal. We can conclude that TH-BPSK is a good choice for UWB application, but it doesn't mean that TH-PPM is not usable. It can be used in many other applications. Additionally, BPSK is able to delete spectral lines which, if existing, could interfere with conventional radio systems. The TH-BPSK receiver detects the two signal polarities (1 and -1). But for the TH-PPM case, we have only one phase to detect which may provide more error with noise.

\section{CONCLUSION}

In this paper we have proposed and analyzed bit error probability performance of TH-BPSK UWB multiple access communication in AWGN channel. We have provided a characteristic mathematical method to calculate the BER. Then, we validate our analytical approach by simulation and comparing with the results found for the TH-PPM case.

From this study, we can conclude that the UWB TH-BPSK system model outperforms UWB TH-PPM for all values of SNR. This result confirms other results find by other authors using different analytical approaches. We can consequently choose the TH-BPSK model as the suitable scheme for UWB communication system design. A natural extension of this work is to include propagation over multipath channels. The proposed analytical approach presented in this article can be extended further to other UWB modulation schemes like TH-OOK. It may be possible in future research to study the performance of TH M-ary Phase Shift Keying (TH-MBPSK) and M-ary Pulse Position Modulation (TH-MPPM).

\section{REFERENCES}

[1] M. Hizem and R. Bouallegue, "Analytical Probability of error in TH-PPM and TH-PAM Ultra Wideband Systems," IEEE ICECS on Electronics, Circuits and Systems, pp. 930-933, September 2008.

[2] P. Saad, C. Botteron, R. Merz, and P.-A. Farine, "Performance comparison of UWB impulse-based multiple access schemes in indoor multipath channels," in proc. 5th Workshop on Positioning, Navigation and Communication (WPCN'08), Hannover, Germany, pp. 89-94, March 2008.

[3] R. A. Scholtz, "Multiple access with time-hopping impulse modulation," IEEE Military Communications Conf., pp. 11-14, 1993.

[4] M. Z. Win and R. A. Scholtz, "Impulse radio: How it works," IEEE Commun. Lett., vol. 2, pp. 36-38, Feb. 1998.

[5] A. Taha and K. M. Chugg, "A theoretical study on the effects of interferenceon UWB multiple access impulse radio," in Proc. Asilomar Conf. Signals, Systems, Computers, pp. 728-732, Nov. 2002.

[6] B. Hu and N. C. Beaulieu, "Accurate evaluation of multiple-access performance in TH-PPM and TH-BPSK UWB systems," IEEE Trans. Commun., vol. 52, no. 10, pp. 1758-1766, Oct. 2004.
[7] Z. Chen, Y. P. Zhang, A. Q. Hu, and T.-S. Ng, "Bit-error-rate analysis of UWB radio using BPSK modulation over inter-chip radio channels for wireless chip area networks," IEEE Transactions on Wireless Communications, vol. 8, no. 5, May 2009.

[8] G. Durisi and S. Benedetto, "Performance evaluation and comparison of different modulation schemes for UWB multiaccess systems," IEEE Int. Conf. Communications, Anchorage, AK, pp. 2187-2191, May 2003.

[9] H. Q. Quach, Narrowband Interference Detection and Mitigation for Indoor Ultra-Wideband Communication Systems, PhD. dissertation, Dept. Elect. Eng., Saskatchewan Univ., Canada, 2006.

[10] I. Hosseini and N. C. Beaulieu, "Bit error rate of TH-BPSK UWB receivers in multiuser interference," IEEE Transactions on Wireless communications, vol. 8, no. 10, October 2009.

[11] G. Durisi and S. Benedetto, "Performance evaluation of TH-PPM UWB systems in the presence of multiuser interference," IEEE Commun. Lett., vol. 7, pp. 224-226, May 2003

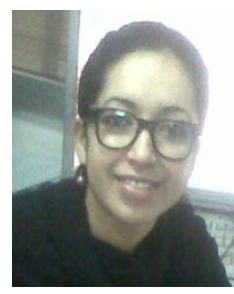

Chihi Houda was born in Tunis, Tunisia. She received the M.S degree in Electronics in 2007 from the Higher School of Engineering Sciences (ESSTT), Tunisia and the M.Sc. degree in Telecommunications in 2009 from the National Engineer School of Tunis (ENIT), Tunisia. She is currently working toward the Ph.D. Degree in Telecommunication systems at the High School of Telecommunication of Tunis (SUP'com) in the Laboratory research of Innovation of Communication and Cooperative Mobiles (Innov'COM), Tunisia. His current research interests include Wireless Communication, CDMA,MBOFDM and Ultra Wideband Systems.

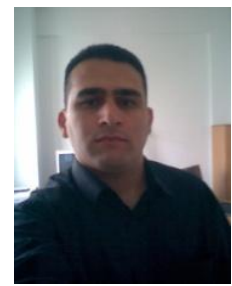

Hizem Moez received the M.S degree in Electronics in 2004 from the Science Faculty of Tunis (FST), Tunisia, the M.Sc. degree in Telecommunications in 2006 and the Ph.D. Degree in Telecommunications in 2011 from the National Engineer School of Tunis (ENIT), Tunisia. Since September 2011, he was an associate professor in the High Institute of Applied Sciences and Technologies (ISSAT), and has taught courses in digital Transmission, mobile and satellite Communications. He is currently working toward the Hd.R. degrees in Telecommunication at the High School of Telecommunication of Tunis (SUP'com) in the Laboratory research of Innovation of Communication and Cooperative Mobiles (Innov'COM), Tunisia. His current research interests include Wireless systems, Modulation formats, Ultra Wideband Systems, and Cooperative and Cognitive radio.

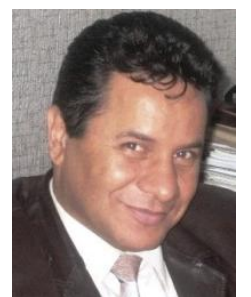

Bouallegue Ridha received the Ph.D degrees in electronic engineering from the National Engineering School of Tunis. In Mars 2003, he received the Hd.R degrees in multiuser detection in wireless communications. From September 1990 he was a graduate Professor in the higher school of communications of Tunis (SUP'COM), he has taught courses in communications and electronics. From 2005 to 2008 , he was the Director of the National engineering school of Sousse. In 2006, he was a member of the national committee of science technology. Since 2005, he was the Innov'COM laboratory research in telecommunication Director's at SUP'COM. From 2005, he served as a member of the scientific committee of validation of thesis and Hd.R in the higher engineering school of Tunis. His current research interests include wireless and mobile communications, OFDM, space-time processing for wireless systems, multiuser detection, wireless multimedia communications, and CDMA systems. 